\title{
Planetary Nebulae as Tracers of Chemical Evolution in External Galaxies
}

\author{
Marshall L. McCall \\ York University, Department of Physics and Astronomy, 4700 Keele \\ Street, Toronto, Ontario, Canada M3J 1 P3 \\ mccall@yorku.ca \\ Michael G. Richer \\ Observatorio Astronómico Nacional, Instituto de Astronomía, UNAM, \\ P.O. Box 439027, San Diego, CA, U.S.A. 92143-9027 \\ richer@astroscu.unam.mx
}

\begin{abstract}
Thanks to recent advances in instrumentation, metal abundances now can be measured routinely for PNe situated in galaxies beyond the Magellanic Clouds. Consequently, PNe are becoming a valuable tool for studying the chemical evolution of external galaxies, especially those which are not forming stars. Here we review problems and progress in this exciting nascent field of interdisciplinary research.
\end{abstract}

\section{Introduction}

Until the mid-1990s, planetary nebulae (PNe) languished in the shadows of HII regions and population syntheses as probes of the chemical evolution of galaxies beyond the Magellanic Clouds. This is because few galaxies were near enough for spectroscopy of PNe to be feasible, and few PNe could be observed adequately in any one galaxy. However, with the advent of multiplexing spectrographs, it has become possible to acquire spectra of a plethora of extragalactic PNe sufficiently deeply to detect the most important temperature and density diagnostics needed to determine abundances reliably. With 4-meter telescopes, quality measurements have already been made out to the distance of M31 and its satellites, and limited spectroscopy has even been performed out as far as Centaurus A at $4 \mathrm{Mpc}$ (Walsh et al. 1999). With the 8-meter generation, it should be possible to measure abundances out to at least $10 \mathrm{Mpc}$. Fundamentally, it is the growth in the sample of galaxies probed by PNe which is facilitating advances in our understanding of how galaxies evolve.

In this paper, research into $\mathrm{PNe}$ which is relevant to studying the evolution of galaxies is reviewed. The focus is on galaxies beyond the Magellanic Clouds, but by necessity inferences drawn from studies of the Milky Way and the Clouds will be made to demonstrate how PNe may be used to probe galaxies. PNe are evaluated as tracers of evolution, not as causes of evolution. Products arising from nucleosynthesis in the progenitors are regarded as contaminants which compromise our ability to study the history of galaxies. 


\section{Galaxy Basics}

Galaxies take many forms, but fundamentally they are comprised of two kinds of stellar systems. Elliptical galaxies, bulges of spiral galaxies, and dwarf spheroidal galaxies (dSphs) all fall into the category of dynamically hot systems (DHSs), in which a significant fraction of the kinetic energy of stars is randomly directed (Bender, Burstein, \& Faber 1992). Such systems are also notable for being severely depleted in gas. Consequently, they host little or no star formation today. Disks, which are found in spiral and irregular galaxies, are believed to have been created during a phase of dissipational collapse. Thus, they are dynamically cold systems (DCSs) in which motions are predominantly ordered, except in the least luminous dwarf irregulars. Most have gas in abundance, and star formation is generally active, although in dwarfs it may be episodic.

\section{Why PNe are Useful}

HII regions are the principal tracers of enrichment in DCSs. They are especially important because they provide information about oxygen, an element whose nucleosynthetic origins and dispersal appear to be simple. However, abundances in DHSs have traditionally been gauged from the integrated spectrum of starlight. Iron abundances result, but they are uncertain owing to the difficulty of disentangling metallicity and age. Furthermore, efforts to study connections between DHSs and DCSs are compromised by lack of knowledge about $\mathrm{O} / \mathrm{Fe}$.

PNe are found in both DHSs and DCSs. In that sense, they are the "missing link" which, with the support of HII regions, can facilitate comparisons of DHSs and DCSs. They provide the only means of studying the abundances of light elements in DHSs at distances where spectroscopy of individual stars is impossible. Considering the importance of oxygen to studies of chemical evolution, they have the potential to revolutionize research into the history of DHSs.

\section{The Process of Determining Abundances}

Abundances of metals in nearby PNe used to be primarily determined from available diagnostics of temperature, density, and ion fractions with the aid of ionization correction factors (ICFs) derived either empirically or with the aid of models (e.g., Kingsburgh \& Barlow 1994). More recently, driven by deeper and deeper spectra, photoionization models have been brought to bear upon the problem, the objective being to fit simultaneously all available data. Spectra of PNe beyond the Magellanic Clouds are reminiscent of those acquired in the earliest days of galactic research. Only the strongest lines are detectable, and typically only one index of temperature ([O III $] \lambda 4363)$ and one index of density ([S II $] \lambda \lambda 6716,6731)$ are measurable. Furthermore, nebulae are unresolved and central stars are too faint to be seen. Consequently, models are horribly unconstrained, and there is no reason to prefer resulting abundances over ICF values (see Péquignot 1997 for a strong dose of realism). Jacoby and Ciardullo (1999) show that ICF and model abundances for PNe in M31 agree, but considering that results from the two methods are determined by the same lines, this is no surprise. The worst thing about model abundances is that there is never enough 
information provided for independent researchers to reproduce them. Thus, as samples of extragalactic PNe spectra grow, systematic differences between modeling methods can lead to inhomogeneities in abundance collections which can be detrimental to studies of chemical evolution. A major advantage of ICF abundances is that uncertainties are directly quantifiable in terms of errors in line strengths. Furthermore, they are reproducible, and readily modifiable as knowledge about ICFs and zonal variations in temperature and density grow. Péquignot et al. (2000a) correctly point out that the true strength of models is in differential analyses of objects with many observational constraints.

For many years, there have been doubts about abundances founded upon forbidden lines, nurtured initially by differences between Balmer jump and auroral line temperatures and the discrepancy between $\mathrm{O} / \mathrm{H}$ in Orion and the Sun, then recently by the discovery that metal recombination lines in $\mathrm{PNe}$ require that abundances be 2 to 10 times higher. Thankfully, two recent discoveries appear to lay these doubts to rest. First, Allende Prieto, Lambert \& Asplund (2001) have shown that the primary diagnostic of $\mathrm{O} / \mathrm{H}$ in the Sun is blended with a nickel line. After compensation, $\mathrm{O} / \mathrm{H}$ is consistent with Orion without any correction for temperature fluctuations (Peimbert 1967). Second, Péquignot et al. (2000b; see also Liu, these proceedings) have presented compelling evidence that the low Balmer jump temperature and high recombination line abundances are a consequence of small inclusions of very cold metal-rich clumps. It now appears that the strengths of forbidden lines in PNe really are a consequence of the temperature indexed by forbidden line ratios, and that derived abundances really do reflect the composition of the bulk of the nebular mass.

\section{What a Single PN Measures}

To be able to use PNe to probe the history of galaxies, it is necessary to presume that the abundance of at least one element reflects conditions in the interstellar medium (ISM) at the time of formation of the progenitor. Unfortunately, during the evolution of 1 to $8 \mathrm{M}_{\odot}$ stars on the giant branch and asymptotic giant branch (AGB), newly synthesized material can be dredged up by convection in the envelope, significantly altering abundances of helium, carbon, and nitrogen in the surface layers (Lattanzio \& Boothroyd 1997; Boothroyd \& Sackmann 1999; Leisy \& Dennefeld 1996; Henry, Kwitter, \& Bates 2000).

Of greatest significance to studies of the chemical evolution of galaxies is what happens to readily measurable $\alpha$-process elements, especially oxygen and neon, produced predominantly in short-lived stars (see below). Standard models indicate that for $M<1.3 \mathrm{M}_{\odot}$ (as in DHSs), $\mathrm{O} / \mathrm{H}$ and $\mathrm{Ne} / \mathrm{H}$ in the envelope should be largely unaffected by AGB evolution (Marigo, Bressan, \& Chiosi 1996). However, during the thermally pulsing phase of AGB evolution (third dredgeup), convection develops in the zone between the hydrogen and helium burning shells. Herwig (2000) has found that if the convection "overshoots" into the core, significant amounts of ${ }^{16} \mathrm{O}$ can be mixed into the inter-shell, some of which may be convected to the surface. Such mixing may also be promoted by rotation (Langer et al. 1999).

Observationally, there is little evidence of oxygen contamination in PNe. In the LMC, SMC, M32, and the bulge and disk of the Milky Way and M31 
there is a tight coupling between $\mathrm{Ne} / \mathrm{H}$ and $\mathrm{O} / \mathrm{H}$ which matches that observed for HII regions (Henry 1989; Stasińska, Richer, \& McCall 1998), implying that both neon and oxygen are produced by the same kinds of stars. Also, the radial distribution of $\mathrm{O} / \mathrm{H}$ derived for Type II PNe across the disk of the Milky Way agrees precisely in slope, level, and dispersion with that of HII regions (Maciel \& Quireza 1999; see also Peimbert \& Carigi 1998). On the other hand, a differential theoretical analysis of two PNe in the Sagittarius dSph (Péquignot et al. 2000a) suggests that $\mathrm{Ne} / \mathrm{O}, \mathrm{S} / \mathrm{O}$, and $\mathrm{Ar} / \mathrm{O}$ differ by $3.5,1.8$, and $1.4 \sigma$, respectively, with the lower values observed in the object with the higher carbon abundance. Comparing $\mathrm{Ne} / \mathrm{O}$ with $\mathrm{HII}$ regions, $\mathrm{O} / \mathrm{H}$ in $\mathrm{PNe}$ with low-mass progenitors may be enhanced by around $20 \%$ ( $0.1 \mathrm{dex})$ by AGB evolution. If systematic, this could lead models of galactic chemistry to over-estimate the yield of oxygen.

\section{What an Ensemble of PNe Measures}

Measurements of the bulk chemical properties of stars and/or gas in a system are required to extract information about history from models of chemical evolution. PNe are merely the "tip of the iceberg", so to be able to employ them to study evolution it is crucial to evaluate how the abundances for an ensemble are related to the properties of the system as a whole.

Targets for spectroscopy are initially selected on the basis of brightness in [O III] $\lambda 5007$. Consequently, if metallicity were the only variable, there might be a bias towards the most metal-rich objects. However, the requirement that a diagnostic of temperature be detectable normally restricts abundance determinations to objects with detectable [OIII] $\lambda 4363$, which should be the least enriched owing to their higher temperatures. In part, at least, the two selection effects ought to cancel (see also Jacoby \& Ciardullo 1999). So far, there is only circumstantial evidence supporting this argument, in that the mean abundance of oxygen derived by Richer \& McCall (2002) for PNe in the bulge of M31 is consistent with that derived by Richer, McCall, \& Stasińska (1998) from a sample with significantly fewer detections of [O III] $\lambda 4363$.

Probably the most important factor which must be accommodated in interpreting abundances from $\mathrm{PNe}$ is the distribution of ages of the progenitors. Richer, McCall, \& Arimoto (1997) investigated the chemical properties of ensembles theoretically by tracking the formation and evolution of PNe during the development of model galaxies via various star formation scenarios, but with self-consistent treatments of chemical evolution. The systems were assumed to be closed. The models predict a $g a p$ of up to 0.3 dex between $\mathrm{O} / \mathrm{H}$ in the ISM (in the case of DHSs, at the time star formation ceased). and the mean value of $\mathrm{O} / \mathrm{H}$ of $\mathrm{PNe}$ within 1 mag of the peak of the luminosity function. The width of the gap grows with the metallicity. What the models make clear is that the interpretation of abundances from ensembles of unresolvable PNe in star-forming systems is not easy. The problem may be compounded by self-enrichment in more massive objects. Simpler to understand may be systems which formed the bulk of their stars long ago, i.e. DHSs. PNe observed today come from progenitors occupying a narrow range of low masses. Consequently, many generations of stars are represented in any magnitude-limited ensemble, and self-enrichment is unlikely to be important. The number of PNe from any generation should 
be roughly proportional to the mass of the generation, so at first glance the chemical properties of the ensemble ought to be representative of those of all stars in the system. In fact, closed models of chemical evolution predict that the gap between the mean value of $\mathrm{O} / \mathrm{H}$ of the stars and that of the ISM should rise with metallicity, just as predicted for the gap between the mean value of $\mathrm{O} / \mathrm{H}$ of the brightest PNe and the ISM. Thus, the ratio of $\mathrm{O} / \mathrm{H}$ for the brightest $\mathrm{PNe}$ to $\mathrm{O} / \mathrm{H}$ in the stars should be relatively constant, if not unity. Although no models have yet been made which test the constancy of the ratio, which is really the most important issue for studies of chemical evolution, the models of Richer et al. (1997) hint that it may not be unity. Likewise, observations of PNe in the bulges of M31 and the Milky Way (Jacoby \& Ciardullo 1999; van de Steene and Jacoby, these proceedings) suggest that the mean abundance of oxygen may be lower for fainter PNe (in fact, as predicted by Richer et al. 1997). However, the mean for the sample employed by Stasińska et al. (1998) is consistent with measurements of $\mathrm{O} / \mathrm{H}$ in bulge giants (McWilliam \& Rich 1994).

\section{The Measurements}

In metal-rich DCSs, HII regions are so cool that metallicities must be founded upon models. PNe may be able to play a significant role in checking abundances if the abundance gap can be evaluated reliably. Even though this wasn't their intent, Jacoby and Ford (1986) found that $\mathrm{O} / \mathrm{H}$ for a PN in the disk of M31 was consistent within errors with the abundance gauged for a nearby HII region from $R_{23}$ (see Pagel et al. 1979; McCall, Rybski, \& Shields 1985). However, no comparison has been made at the cooler temperatures characteristic of most of the HII regions in giant spirals.

Recently, the majority of abundance measurements have been made for PNe in DHSs. This is because of the much more significant role that PNe can play in understanding the evolution of such systems.

There has been an ongoing debate about whether dwarf spheroidal galaxies (dSphs) are related to dwarf irregular galaxies (dIs), since the two kinds of systems are structurally similar. Assuming that $\mathrm{O} / \mathrm{Fe}$ is the same in dSphs as in dIs, Skillman, Kennicutt, \& Hodge (1989) argued in favour of a linkage on the grounds that they display the same relationship between $\mathrm{O} / \mathrm{H}$ and luminosity. By studying PNe in NGC 185 and NGC 205, Richer \& McCall (1995) and Richer et al. (1997) proved that $\mathrm{O} / \mathrm{Fe}$ is significantly higher in dSphs than in the SMC and LMC. Thus, dSphs must have formed their stars much faster than dIs, and are more likely to be related to star-bursting blue compact dwarfs (BCDs).

It has long been known that the metallicity of a DHS, as judged from $\mathrm{Fe} / \mathrm{H}$, is correlated with mass (e.g., Dekel \& Silk 1986; Bender, Burstein, \& Faber 1993). The correlation is believed to be evidence that gas was lost via a wind once supernovae raised the internal energy up to a level comparable to the binding energy (Larson 1974). In this context, since oxygen comes from the same massive stars responsible for much of the energy injection, the abundance of oxygen should strongly correlate with the dispersion of stellar velocities, which gauges the depth of the potential well. Indeed, observations of PNe in Sagittarius (Walsh et al. 1997), Fornax (Maran et al. 1984; Walsh et al. 1997), NGC 185 (Richer \& McCall 2002), NGC 205 (Richer \& McCall 2002), M32 (Richer \& 
McCall 2002; Richer, Stasińska, \& McCall 1999), and the bulges of M31 (Richer et al. 1999; see also Jacoby \& Ciardullo 1999) and the Milky Way (Ratag et al. 1997; Webster 1988; Aller \& Keyes 1987; see also Cuisinier et al. 2000) show just such a trend, especially once $\mathrm{O} / \mathrm{Fe}$ is used to account for the additional energy contributed by Type I supernovae (Richer \& McCall, these proceedings).

Many DHSs show gradients in line strengths with radius, leading to the belief that there are gradients in abundances, just as there are across DCSs. Besides being important for assessing theories which try to explain DHS formation in terms of mergers, gradients may artificially inflate abundance dispersions, which are important constraints on models of chemical evolution. Across M32, the $11 \mathrm{PNe}$ with measured temperatures do not display any significant gradient in $\mathrm{O} / \mathrm{H}$ out to 8.5 effective radii (Richer \& McCall 2002). However, M32 is a dwarf. It would be profitable to direct future research towards giant DHSs displaying strong gradients in absorption line strengths.

\section{How Measurements are Linked to Evolution}

Models of chemical evolution can predict the metal abundance in the ISM, the mean metallicity of stars, and the dispersion in the metallicity of stars as a function of the fraction of the mass of a system remaining as gas. The problem with even the simplest models, though, is that there are normally more unknowns than variables. For example, suppose $\mathrm{PNe}$ constrain the mean and dispersion in $\mathrm{O} / \mathrm{H}$ in $n$ DHSs. There are $2 n$ observations, but allowing for the possibility of flows of gas during enrichment, there are $2 n+1$ unknowns (1 yield, $n$ gas fractions, and $n$ flow rates). Nevertheless, a fundamental prediction is that if a system evolves in isolation, then the gas fraction must decline as the abundance of an instantaneously recycled product of nucleosynthesis rises. In DHSs, the gas fraction is a constant, namely zero. Yet, the abundance of oxygen grows in a systematic way with mass. The logical conclusion is that evolution was not closed, and that star formation stopped because of gas loss, with less massive systems losing greater fractions of their gas. The supernovae-driven wind model is a compelling explanation, because the temperature of the gas when a wind develops is close to the virial temperature, which is linearly proportional to the potential of the system. Systems with deeper potential wells are able to form stars longer and become more enriched before the temperature of the gas reaches the virial temperature.

The supernovae-driven wind adds a dynamical constraint which breaks the degeneracy of models for the evolution of instantaneously recycled elements (McCall, Richer, \& Stasińska 1998). The lack of such a well-defined constraint is a principal factor limiting interpretations of abundances in DCSs. Suppose that a DHS has a stellar mass $M_{*}$ and a gas mass $M_{g}$ at the time of onset of the wind, $t_{\text {wind }}$. Because the build-up of internal energy was caused mainly by Type II supernovae, $M_{*}$ is a measure of the internal energy, which through the virial theorem is related to the potential energy of the gas. Since the stars seen today have virialized in response to a potential like that felt by the gas at $t_{\text {wind }}$, it is easy to show that $M_{*} / M_{g} \propto g K_{\text {now }}$, where $K_{\text {now }}$ is the internal energy observable today in the form of random and rotational motions of stars, and $g$ is a factor which takes into account the possibility that the potential felt by the 

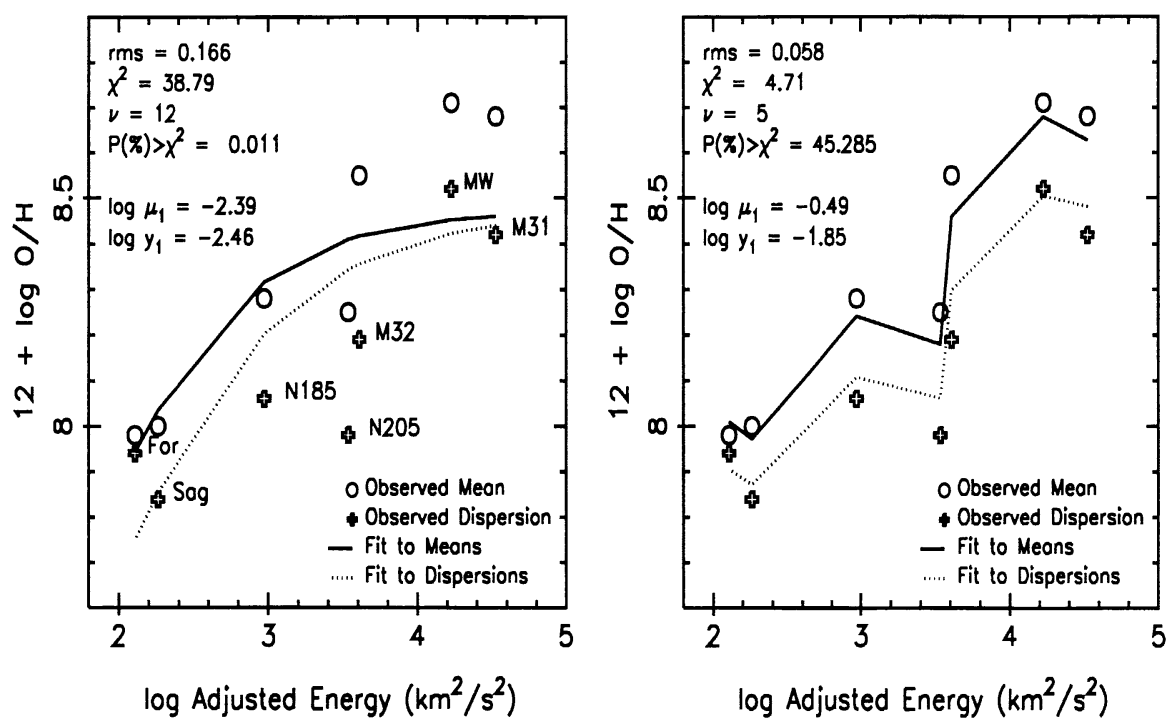

Figure 1. Linear means and dispersions of $\mathrm{O} / \mathrm{H}$ in seven DHSs. Left: Fits assume closed evolution prior to a supernovae-driven wind plus a potential governed by dark matter. Right: Fits assume that there was slow outflow prior to the wind and that the potential felt by the nucleogenetic pool was set by baryons.

baryons adjusted after mass loss. For a potential controlled by dark matter, $g=1$. However, for a system whose mass is predominantly baryonic, or whose baryons don't feel the potential of dark matter (as might be the case if the dark matter halo is very extended relative to the baryons), then $g=1 /(1-2 \mu)$, where $\mu$ is the gas fraction (Hills 1980). With a choice for $g$, the gas fraction of any galaxy can be expressed in terms of the gas fraction of one. Thus, for $n$ DHSs, there are $n+2$ unknowns ( 1 yield, 1 gas fraction, $n$ flow rates). With $2 n$ observations, it is possible to solve for the unknowns exactly if $n \geq 2$.

Figure 1 shows for seven DHSs how the mean and dispersion of $\mathrm{O} / \mathrm{H}$ varies with the kinetic energy per unit mass of stars. Models of chemical evolution naturally predict the linear mean and dispersion; the quantities plotted are the logarithms of these linear values, augmented by 12 . Stellar energies per unit mass, derived from velocity dispersions and rotational velocites, have been reduced by a factor related to $\mathrm{O} / \mathrm{Fe}$ to account for the delayed heating by Type I supernovae (Richer et al. 1998). The supernovae-driven wind constraint predicts that the relationship between $\mathrm{O} / \mathrm{H}$ and this adjusted energy ought to mimic the relationship between $\mathrm{O} / \mathrm{H}$ and $M_{*} / M_{g}$.

The figure shows that both the mean and dispersion in $\mathrm{O} / \mathrm{H}$ tends to grow with the adjusted energy of the system. The deviant points are due to NGC 205. Typical errors in the plotted quantitites are about 0.1 dex, but data for Fornax and Sagittarius are more uncertain. They contain so few $\mathrm{PNe}(1$ and 2 , respectively) that the dispersions have been adopted to be the same as for $\mathrm{Fe} / \mathrm{H}$. 
Furthermore, since their PNe may be from a younger population of stars, mean values of $\mathrm{O} / \mathrm{H}$ have been adjusted downwards by amounts equal to the dispersions in $\mathrm{Fe} / \mathrm{H}$ (see Walsh et al. 1997 for a contrary view).

Figure 1 (left) also displays the simultaneous fits to the means and dispersions which result when it is assumed that evolution before $t_{\text {wind }}$ was closed. The model is unable to reproduce the large separation between the dispersion and the mean in the largest DHSs, which is a characteristic typical of systems in which chemical cycling is less advanced. It also cannot explain NGC 205. To arrive at acceptable fits for all systems (Figure 1, right), it is necessary to allow outward flows of gas before $t_{\text {wind }}$ unique to each galaxy. Furthermore, potentials must be allowed to adjust to the loss of gas, with the potential gain factor being greater for small systems compared to large, as prescribed if baryons control the potential felt by the nucleogenetic pool. The resulting yield of oxygen is four times greater than observed locally. Owing to slow gas loss prior to $t_{\text {wind }}$, the models suggest that dwarfs such as Fornax began with as much as 10 times more mass than is present in stars today. The bulge of the Milky Way started out $40 \%$ more massive than it is now, losing the bulk of its gas at $t_{\text {wind }}$.

\section{The Future}

In DCSs, HII regions will likely remain the preferred means of studying chemical evolution, with extragalactic PNe playing an increasingly visible role in support. However, PNe have the potential to revolutionize the studies of DHSs, provided that some key questions are resolved. Most important, both observations and theory must be expanded to elucidate precisely what it is that an ensemble of PNe probes, i.e. how closely chemical properties of [O III] $\lambda 4363$-limited subsets of $[\mathrm{O}$ III] $]$ 5007-selected samples resemble those of the bulk of the stars. The most profitable arena for investigations is the bulge of the Milky Way, where $\mathrm{O} / \mathrm{H}$ can be derived for both stars and $\mathrm{PNe}$, and for which proximity makes possible the determination of ages of PNe (Dopita et al. 1997). Models of the chemical evolution of DHSs will remain uncertain until the sample is expanded to include giant ellipticals, which will shift weight away from dwarfs whose data are compromised by poor statistics, and thereby improve the confidence with which the yield of oxygen is determined. With luck, PNe one day will enable evaluation of the systematics of gas loss by DHSs, and a determination of both the amount and composition of gas injected by all such objects into the intergalactic medium.

\section{References}

Allende Prieto, C., Lambert, D. L., \& Asplund, M. 2001, ApJ, 556, L63

Aller, L. H. \& Keyes, C. D. 1987, ApJS, 65, 407

Bender, R., Burstein, D., \& Faber, S. M. 1992, ApJ, 399, 462

Bender, R., Burstein, D., \& Faber, S. M. 1993, ApJ, 411, 153

Boothroyd, A. I. \& Sackmann, I.-J. 1999, ApJ, 510, 232

Cuisinier, F., Maciel, W. J., Köppen, J., Acker, A., \& Stenholm, B. 2000, A\&A, 353, 543

Dekel, A., \& Silk, J. 1986, ApJ, 303, 39 
Dopita, M. A., Wood, P. R., Meatheringham, S. J., Vassiliadis, E., Bohlin, R. C., Ford, H. C., Harrington, J. P., Stecher, T. P., \& Maran, S. P. 1997, in IAU Symp. 180, Planetary Nebulae, eds. H. J. Habing \& H. J. G. L. M. Lamers (Dordrecht: Kluwer), 417

Henry, R. B. C. 1989, MNRAS, 241, 453

Henry, R. B. C., Kwitter, K. B., \& Bates, J. A. 2000, ApJ, 531, 928

Herwig, F. 2000, A\&A, 360, 952

Hills, J. G. 1980, ApJ, 225, 986

Kingsburgh, R. L., \& Barlow, M. J. 1994, MNRAS, 271, 257

Jacoby, G. H., \& Ciardullo, R. 1999, ApJ, 515, 169

Jacoby, G. H., \& Ford, H. C. 1986, ApJ, 304, 490

Langer, N., Heger, A., Wellstein, S., \& Herwig, F. 1999, A\&A, 346, L37

Larson, R. B. 1974, MNRAS, 169, 229

Lattanzio, J. C., \& Boothroyd, A. I. 1997, in Astrophysical Implications of the Laboratory Study of Pre-Solar Materials, eds. T. J. Bernatowicz \& E. Zinner (Woodbury, NY: AIP), 85

Leisy, P., \& Dennefeld, M. 1996, A\&AS, 116, 95

Maciel, W. J., \& Quireza, C. 1999, A\&A, 345, 629

Maran, S. P., Gull, T. R., Stecher, T. P., Aller, L. H., \& Keyes, C. D. 1984, ApJ, 280, 615

Marigo, P., Bressan, A., \& Chiosi, C. 1996, A\&A, 313, 545

McCall, M. L., Richer, M. G., \& Stasińska, G. 1998, in ASP Conf. Series Vol. 147, Abundance Profiles: Diagnostic Tools for Galaxy History, eds. D. Friedli, M. Edmunds, C. Robert, \& L. Drissen (San Francisco: ASP), 259

McCall, M. L., Rybski, P. M., \& Shields, G. A. 1985, ApJS, 57, 1

McWilliam, A., \& Rich, R. M. 1994, ApJS, 91, 749

Pagel, B. E. J., Edmunds, M. G., Blackwell, D. E., Chun, M. S., \& Smith, G. 1979, MNRAS, 189, 95

Peimbert, M. 1967, ApJ, 150, 825

Peimbert, M., \& Carigi, L. 1998, in ASP Conf. Series Vol. 147, Abundance Profiles: Diagnostic Tools for Galaxy History, eds. D. Friedli, M. Edmunds, C. Robert, \& L. Drissen (San Francisco: ASP), 88

Péquignot, D. 1997, in IAU Symp. 180, Planetary Nebulae, eds. H. J. Habing \& H. J. G. L. M. Lamers (Dordrecht: Kluwer), 167

Péquignot, D., Walsh, J. R., Zijlstra, A. A., \& Dudziak, G. 2000a, A\&A, 361, L1

Péquignot, D., Amara, M., Liu, X.-W., Morisset, C., Barlow, M. J., \& Storey, P. J. 2000b, in Ionized Gaseous Nebulae (Mexico City), 46

Ratag, M. A., Pottasch, S. R., Dennefeld, M., \& Menzies, J. 1997, A\&AS, 126, 297

Richer, M. G., \& McCall, M. L. 2002, in preparation

Richer, M. G., \& McCall, M. L. 1995, ApJ, 445, 642

Richer, M. G., McCall, M. L., \& Arimoto, N. 1997, A\&AS, 122, 215

Richer, M. G., McCall, M. L., \& Stasińska, G. 1998, A\&A, 340, 67

Richer, M. G., Stasińska, G., \& McCall, M. L. 1999, A\&AS, 135, 203

Skillman, E. D., Kennicutt, R. C., \& Hodge, P. W. 1989, ApJ, 347, 875

Stasińska, G., Richer, M. G., \& McCall, M. L. 1998, 336, 667

Walsh, J. R., Dudziak, G., Minniti, D., \& Zijlstra, A. A. 1997, ApJ, 487, 651

Walsh, J. R., Walton, N. A., Jacoby, G. H., \& Peletier, R. F. 1999, A\&A, 346, 753

Webster, B. L. 1988, MNRAS, 230, 377 


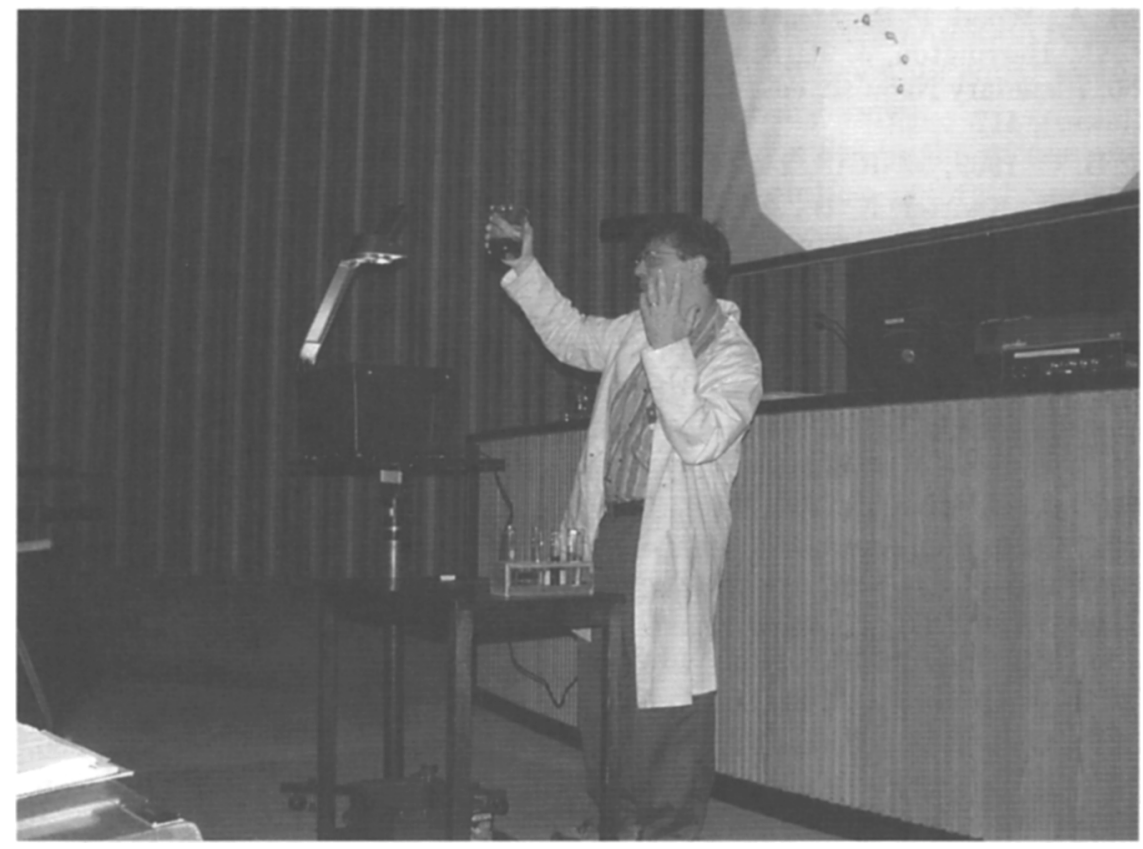

Marshall McCall demonstrates the cycling of metals in galaxies.

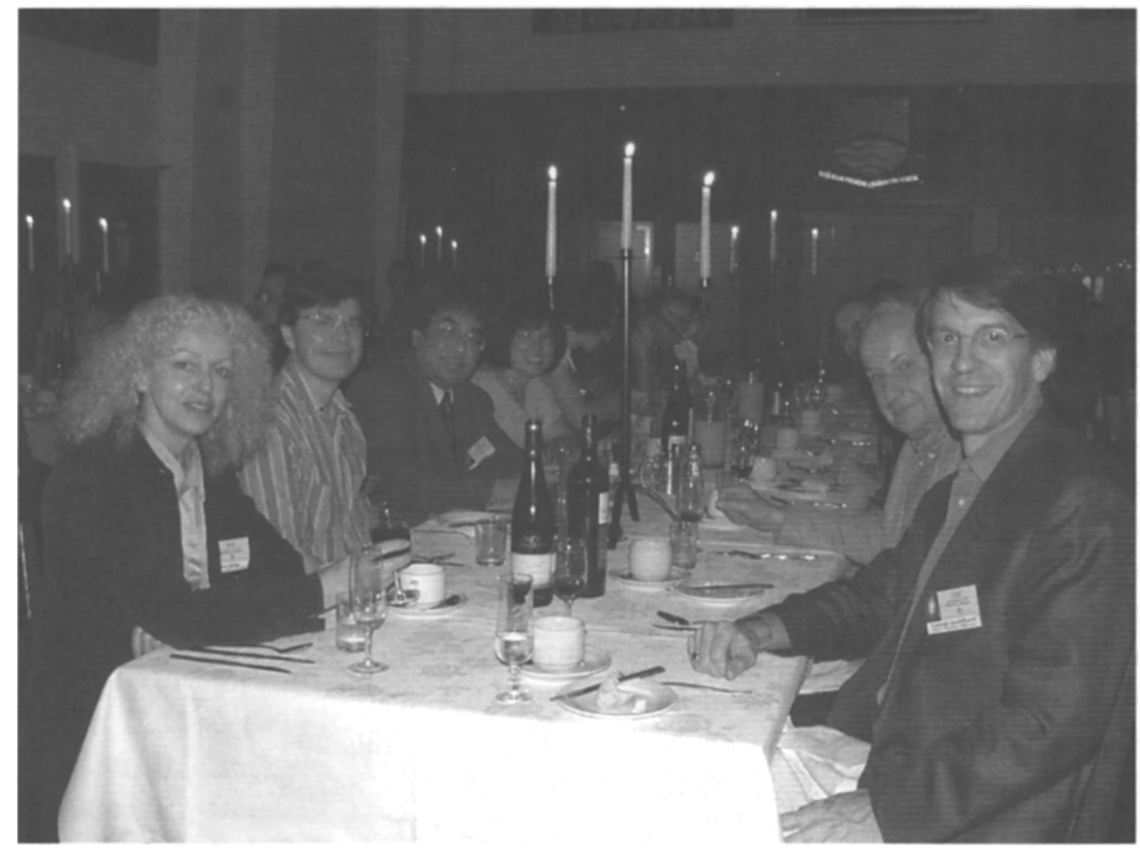

From left to right: Rosina Iping, Marshall McCall, Sun Kwok, Emily Kwok, Harm Habing, and George Sonneborn. 